\title{
ROLE OF SWISS BALL EXERCISES IN REDUCING PAIN, DISABILITY AND IMPROVING MUSCLE ENDURANCE IN PATIENTS WITH MECHANICAL LOW BACK ACHE
}

\author{
Sumit Raghav *, Anshika Singh.
}

Subharti College Of Physiotherapy, Swami Vivekanand Subharti University, Meerut (U.P.), India.

\section{ABSTRACT}

Introduction: Low back pain is a very common health problem associated with considerable disability and costs to society. Low back pain is becoming increasingly common. Approximately $70-90 \%$ of our population suffers from low back pain or has suffered from multiple episodes of low back pain. Pain aggravated by extension, sideflexion, rotation, standing, walking, sitting, and exercise in general, and relieved by changing in position. Many different therapeutic interventions are used in the management of low back pain. Swiss ball exercises have become an important part of the fitness regimen and widely used exercises for low back ache.

Materials and Methods: The study was of an experimental design, with 30 subjects, 13 were female, 17 were male, and all subjects were assigned according to criteria (inclusion \& exclusion) and carried out at physiotherapy OPD of CSS Hospital, Meerut. Disability \& pain were assessed by using the ODI \& VAS score respectively while endurance of abdominal and trunk extensors muscles assessed by using DAET and DEET score. The subjects were reassessed after completion of 6 weeks of intervention. The collected data were of mean and standard deviation of ODI, VAS, DAET and DEET score and has been analyzed using SPSS software. The study was done to find out the efficacy of Swiss ball exercises in reducing pain, disability and improving endurance of muscles (abdominal and trunk) in patients with mechanical low back ache.

Results: The results showed that there were significant difference in pain, disability and muscle endurance with their VAS, ODI score $(p=0.001)$ and DAET, DEET score respectively.

Conclusion: Study concluded that the difference from $1^{\text {st }}$ to $42^{\text {nd }}$ day in VAS, ODI, DEET \& DEET score which shows that swiss ball exercises are effective to decrease pain, disability and improve muscle endurance.

KEY WORDS: MLBA (Mechanical Low Back ache), ODI (Oswestry Disability Index) VAS (Visual Analog Scale), DAET (Dynamic Abdominal Endurance Test) and DEET (Dynamic Extensors Endurance Test).

Address for correspondence: Dr. Sumit Raghav, Subharti College Of Physiotherapy, Swami Vivekanand Subharti University, Meerut (U.P.), India. E-Mail: drsumitraghav@gmail.com

\begin{tabular}{|c|l|}
\hline \multicolumn{3}{|c|}{ Access this Article online } \\
\hline Quick Response code & International Journal of Physiotherapy and Research \\
ISSN 2321- 1822
\end{tabular}

\section{INTRODUCTION}

Low back pain is a very common health problem associated with considerable disability and costs to society. Many different therapeutic interventions are used in the management of low back pain. For clinicians, researchers, and policy makers, it is important to be able to determine the most successful treatment [1].

Low back pain is becoming increasingly common.
Approximately $70-90 \%$ of our population suffers from low back pain or has suffered from multiple episodes of low back pain. Low back pain can inhibit an individual from leading an active life style [2]. In 1990, nearly 15 million office visits took place for mechanical low back pain, ranking this problem as the second most common symptom related reason for seeing a physician Survey suggest that the life time incidence 
of low back pain ranges form $60-90 \%$ within $5 \%$ annual incidence. For person younger than 45 years, mechanical low back pain represents the most common cause of disability than in person aged older than 45 years [3].

It has a prevalence of $60-85 \%$ during an individual lifetime [4] and it may vary according to age, gender, education and occupation. Women's have higher rate of developing low back pain [5].

The lifetime prevalence of low back pain is reported to be as higher as $84 \%$ \& best estimated suggest that the prevalence of chronic low back pain is about $23 \%$ with $11 \%-12 \%$ of population disabled by it. Non specific low back pain caused by the mechanical factor. Bradford Hill' criteria include the occupational sitting, awkward postures, standing \& walking, pushing $\&$, bending and twisting, lifting and carrying were independently causative for low back pain in the population of workers [5].

Pain aggravated by extension, side-flexion, rotation, standing, walking, sitting, and exercise in general, and relieved by changing in position [6]. The strengthening program of core can be used or essential for the prevention of injuries around lower back. The endurance of trunk muscles is low in patients with low back pain as compared to individual without low back pain. The deep trunk muscles (Transverse Abdominis \& Multifidus) responsible for maintaining the stability of spine. It is studied that the poor endurance of trunk muscles may caused strain on structures \& hence causes low back pain [7].

Exercises are commonly prescribed for low back pain by physical therapist. The aims is to abolishing pain, restoring and maintaining full range of motion, improve the strength and endurance of lumbar and abdominal muscles. Jackson and Brown (1983) opinion that exercises will decrease the pain , strengthen muscles, decreased mechanical stress to spinal structures, improves fitness level, prevent injury and improve postures and mobility in patient with low back pain [8].

Aims and Objective: To assess the efficacy of Swiss ball exercises for reducing pain, disability and improving muscle endurance in patient with Mechanical low back ache.

\section{MATERIALS AND METHODS}

Visual Analogue Scale: The visual analog scale is one of the most basic pain measurement tools. It consists of a $10 \mathrm{~cm}$ line. The clinician can measure the place on the line and convert into it a score between 0 to 10 where 0 is no pain and 10 is bad as it could be [9].

Oswestry Disability Index: Oswestry disability index is a good functional scale because it deals with activity of daily living \& therefore is based on the patient response and concerns affecting daily life. It is used to measured patient perceived functional disability e.g., work status, difficulty caring for oneself.

It is most commonly used functional back scale. It is calculated by dividing the total score (1-6) by number of section answered and multiplying by 100 [9].

Dynamic Abdominal Endurance Test: This test checks the endurance of the abdominals. The patient is in supine position with the hip 45 degree and knee at 90 degree and hands at sides. A line of $8 \mathrm{~cm}$ (for over $40 \mathrm{yr}$ of age) or $12 \mathrm{~cm}$ (under $40 \mathrm{yr}$ of age) is drawn distal to the fingers. The patient tucks in the chin and curls the trunk to touch the line with fingers and repeats as many curls as possible using a cadence of 25 repetition/min. This test may also be done as an isometric by assuming the end position and holding it.

The grading for this isometric abdominal test would be as follows:

(Normal) 5. Hands behind neck, until scapulae clear the table (20-30 sec hold).

(Good) 4. Arm crossed over chest, until scapula clear table (15-20sec hold).

(Fair) 3. Arm straight, until scapulae clear table (10-15 sec hold).

(Poor) 2. Arm extended, towards knees, until top of scapula lift from table (1-10 sec hold).

(Trace) 1. Unable to raise more than head off table [10].

Dynamic Extensors Endurance Test: This test is designed to test the strength of iliocostalis lumborum (erector spinae) and multifidus. The patient is placed in prone lying with the hips and iliac crests resting on the end of the exam- 
ining table and the hips and pelvis stabilized with the straps. Keeping the spine straight, the examiner instructs the patient to extend the trunk to neutral and then lower the head to the start position. The patient arm should be crossed at the chest. The cadence is 25 repetition $/ \mathrm{min}$. This test may also be done isometrically, the examiner times how long the patients hold the contraction without pelvic or spinal movement. In this case the patient can start with the hands by the sides, moving the hands behind the head for increasing the difficulty.

The test, if done isometrically (isometric extensor test), would be as follows:

(Normal) 5- With hands clasped behind the head, extends the lumbar spine, lifting the head, chest, and ribs from the floor.(20-30 sec hold).

(Good) 4- With hands at the the side, extends the the lumbar spine, lifting the sternum off the floor (15-20 sec hold).

(Fair) 3-With hands at the side, extends the lumbar spine, lifting the sternum off the

floor.(10-15 sec hold).

(Poor) 2- With hands at the side, extends the lumbar spine, lifting the head off the floor(1-

10 sec hold).

(Trace) 1- Only slight contraction of the muscle with no movement [13].

Hypothesis

Experimental Hypothesis: There is significant effect of Swiss ball exercise to reduce pain, disability and improving muscle endurance in patients with mechanical low back ache.

Null Hypothesis: There is no significant effect of Swiss ball exercise to reduce pain, disability and improving muscle endurance in patients with mechanical low back ache.

Limitation of study: Small sample size, The duration of study is small, research is done only among a particular age group and Only pain, disability and endurance of back extensors and flexors muscles are measured.

Variables

Dependent Variables: VAS score and ODI score, DAET and DEET, This study is an experimental design in nature.
Sample selection: Convenient sample of 30 subjects, according to the inclusion and exclusion criteria, randomly assigned in the study. This study was conducted in physiotherapy OPD of CSS Hospital Subharti University Meerut.

Inclusion Criteria: Age- 30 - 40, Males and females with mechanical low back pain and Pain duration more than 6 weeks.

Exclusion Criteria: Spina bifida, Scoliosis, Spondylosis, Spondylolisthesis, Prolapsed intervertebral disc, Fracture of vertebral column, Ankylosing spondylosis, Pott's spine, Tumours, Radiculopathy, Any spinal surgery, Gynecological cause, Athletes \& gymnasts.

Instrumentation: Mattress, Towel, Moist Heat Pack, Swiss Ball $(65 \mathrm{~cm}, 75 \mathrm{~cm})$ depending on the height of the subjects, Stationary (Pen, Pencil \&Paper), Questionnaire (Oswestry Disability Index) and Consent Form

Protocol: After getting their informed consent the subjects were randomly assigned. It was given to the patient with duration of 15 minutes/ day for 3 days in a week. Warm up exercises were given to the patient for 10 minutes (before swiss ball exercise) to improve tissue extensibility. The protocol included treatment of total six weeks, with three days per week management. The patients included were encouraged to complete the home exercise program such as isometric exercise of back flexors, back extensors and lateral flexors for six weeks period.

Data Analysis: All analysis was obtained using SPSS version 21.0. Demo graphic data of the patients including age and gender were summarized. The dependent variables for the statistical analysis were VAS and ODI. A base line data was taken at the beginning of the study (pre test values) and after the completion of the treatment (post test values) to analyze the difference between the pre and post values; paired t-test was used. A level of 0.05 was used to determine the statistical significance.

\section{RESULTS AND TABLES}

A sample of size 30 was studied individually for VAS, ODI, DAET and DEET score at base line 1st and $42^{\text {nd }}$ day respectively. (Table-1) presents the Mean \& S.D. and standard error of Mean for 
Pre and Post VAS and ODI score. (Table-2) presents the Mean, Standard Deviation for the pre and post DEET and DAET score difference $\left(1^{\text {st }}\right.$ $42^{\text {nd }}$ Day).

The Paired ' $\mathrm{t}$ ' test was applied to find the significant difference between Pre and Post VAS, ODI and DEET, DAET score which shows a significant difference at $1 \%$ level of significance $(P<0.001)$.

The average difference from $1^{\text {st }}$ to $42^{\text {nd }}$ day in VAS and ODI score shown in (Table 3 ) reduce pain and disability.

Table 1: Mean, Standard Deviation \& S.E.M. of VAS score and ODI score.

\begin{tabular}{|c|c|c|c|c|c|}
\hline \multirow{2}{*}{ S.NO } & \multirow{2}{*}{ TIME PERIODS } & \multicolumn{2}{|c|}{ (MEAN \pm S.D.) } & \multicolumn{2}{c|}{ S.E.M } \\
\cline { 3 - 6 } & & VAS & ODI & VAS & ODI \\
\hline $\mathbf{1}$ & \multirow{2}{*}{${\text { AT } 1^{\text {st }} \text { DAY }}^{*}$} & $5.03 \pm 1.13$ & $46.13 \pm 4.34$ & 0.21 & 0.79 \\
\hline $\mathbf{2}$ & ${\text { AT } 42^{\text {nd }} \text { DAY }}$ & $1.23 \pm 1.04$ & $19.23 \pm 1.68$ & 0.19 & 0.31 \\
\hline
\end{tabular}

Table 2: Mean, Standard Deviation \& S.E.M Of Pre \& Post Scores Of Dynamic Abdominal Endurance Test, Dynamic Extensors Endurance Test.

\begin{tabular}{|c|c|c|c|c|c|}
\hline \multirow{2}{*}{ S.NO } & \multirow{2}{*}{$\begin{array}{c}\text { TIME } \\
\text { PERIODS }\end{array}$} & \multicolumn{2}{|c|}{ (MEAN \pm S.D.) } & \multicolumn{2}{|c|}{ S.E.M } \\
\hline & & DAET & DEET & DAET & DEET \\
\hline 1 & AT $1^{\text {st }}$ DAY & $2.37 \quad 0.49$ & 2.570 .50 & 0.09 & 0.09 \\
\hline 2 & AT $42^{\text {nd }}$ DAY & $3.40 \quad 0.50$ & 3.330 .48 & 0.09 & 0.09 \\
\hline
\end{tabular}

Table 3: Paired T - test, Average difference in Mean and standard Deviation of pre and post score of VAS, ODI, DEET and DAET.

\begin{tabular}{|c|c|c|c|c|c|}
\hline & \multicolumn{4}{|c|}{ PAIRED DIFFERENCES } & \multicolumn{1}{|c|}{} \\
\hline VARIABLES & MEAN & S.D & S.E & t-value & p-value \\
\hline PRE TO POST ODI & 26.9 & 3.47 & 0.63 & 42.49 & $<0.001($ sig.) \\
\hline PRE TO POST VAS & 3.8 & 0.66 & 0.12 & 31.32 & $<0.001($ sig.) \\
\hline PRE TO POST DAET & -1.03 & 0.41 & 0.08 & -13.67 & $<0.001($ sig.) \\
\hline PRE TO POST DEET & -0.77 & 0.57 & 0.1 & -7.389 & $<0.001($ sig.) \\
\hline
\end{tabular}

The table above shows that a significant difference is present in Pre \& Post values of ODI, VAS, DAET \& DEET.

Graph 1: Pre \& Post values of Mean of ODI, VAS, DEAT \& DEET.

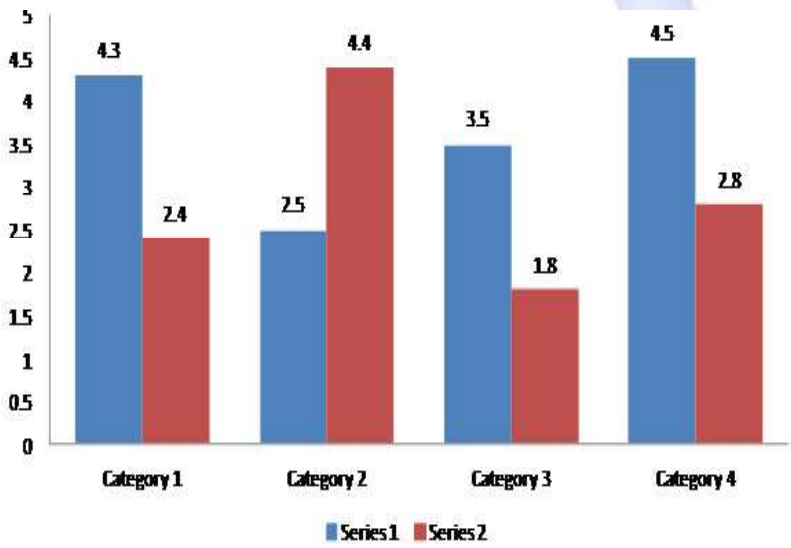

\section{DISCUSSION}

The findings of this study indicated that subjects had significant decrease in pain, disability and improve the endurance of muscles. The data showed that with the use of six weeks protocol there was significant difference between post treatment values of VAS score, ODI score, DAET score and DEET score taken on $42^{\text {nd }}$ day. Thus Behm $\mathrm{G}$ et al, showed that there is evidence exercises performed on unstable (Swiss Ball) surface stressed the musculature and activated the propioception activity \& gain the Stability. Petersen et al concluded that reduction of pain is due to strengthening exercises in patient with Mechanical low back pain. Ludmilla M, CosioLima, Katy L et al (2003) a study "effects of physioball and conventional floor exercises on early phase adaptation in back and abdominals core stability and balance in women" for 5 weeks and the physioball group was found to give significantly greater mean change in EMG flexion and extension activity and greater balance scores [11].

Janessa D.M Drake, Steve L Fischer, Stephen H.M Brown et al in a study " Do Exercise Ball Provide a Training Advantage for trunk extensor Exercises? A biomechanical evaluation" on 8 subjects came with the result that the use of an exercise ball will always create a greater challenge for the musculoskeletal system was not supported by this study. In young, healthy population, there doesn't appear to be any training advantage to performing exercise on a ball versus mat. However, in a rehabilitation scenario, these exercise performed on a ball could reduce low back loading and hence reduce the potential for injury [12].

\section{CONCLUSION}

The study states that the Swiss ball exercises decrease pain, improve endurance (abdominal and trunk muscles), and decrease the functional disability in patients with mechanical low back pain. It is concluded that the swiss ball exercises are effective in reduction of pain and disability and increases the endurance of muscles. Study conclude that the difference from $1^{\text {st }}$ to $42^{\text {nd }}$ day in VAS and ODI score which is shown in 
(Table 3) which shows that swiss ball exercises are effective to decrease pain and disability, improve endurance.

The study shows that the parameters utilized for the technique maneuvers were effective for decreasing pain and disability. This Study supports experimental hypothesis H1.The significant difference was present for VAS score, ODI score as well as DAET score and DEET score.

\section{Conflicts of interest: None}

\section{REFERENCES}

[1]. Nicola Vader Roer, Minimal Clinically Important Change for Pain Intensity, Functional Status, and General Health Status in Patients with Nonspecific Low Back Pain. SPINE 2006;31(5):578-582.

[2]. Michael A. Clark, Alan M.Rusell. Article on LBP; A Functional Perspective 2005, https:// www.performbetter.com/webapp/wcs/stores/ servlet/PB One Piece View ? storeld $=10151$ \& cata$\log I d=10751 \&$ page name $=78$

[3]. Everett C Hills, Stephen Kishner. et al. Mechanical low back pain. http://emedicine.medscape.com/article/310353-overview

[4]. Shankar G, Chauarsia V. Comparative Study of Core Stability Exercises with Swiss Ball in Improving Trunk Endurance. International Journal of Health Sciences \& Research. 2012;2(5).
[5]. Pamela k, levangie, Cynthia c. norkin: structure and function of lumbar spine; a comprehensive analysis, 4th edition, published by jaypee brothers.

[6]. NICE Guideline. Low back pain : early management of persistent non-specific low back pain, may 2009.

[7]. David J. Magee: Orthopedic physical assessment, chapter 9, 5th edition, Published by W.B.Saunders; page no 524

[8]. Jackson. C.P. and Brown M.D. Is there a role for exercise in the treatment of patients with low back pain? Clinical orthopedics and related research, 1983;179:39-45.

[9]. Susan B O'Sullivan \& Thomas J Schmitz: physical rehabilitation, chapter $11,5^{\text {th }}$ edition, published by Jaypee brothers medical publishers Itd, page no. 381.

[10]. David J. Magee: Orthopedic physical assessment, chapter 9, 5th edition, Published by W.B.Saunders; page no 553-54 and 540-44.

[11]. Ludmilla M, Cosio-Lima, Katy L, et al. Effects of Physioball and Conventional Floor Exercises on Early Phase Adaptation In Back and Abdominal Core Stability and Balance in Women. The Journal of Strength and Condition Research; 2002;17(4):721725.

[12]. Drake JD, Fischer SL, Brown SH, Callaghan JP. Do exercise balls provide a training advantage for trunk extensor exercises? A biomechanical evaluation. Journal of manipulative and physiological therapeutics. 2006 Jun 30;29(5):354-62.

How to cite this article:

Sumit Raghav, Anshika Singh. ROLE OF SWISS BALL EXERCISES IN REDUCING PAIN, DISABILITY AND IMPROVING MUSCLE ENDURANCE IN PATIENTS WITH MECHANICAL LOW BACK ACHE. Int J Physiother Res 2017;5(2):19661970. DOI: $10.16965 /$ ijpr.2017.117 